\title{
Fixed Points of Modified F-Contractive Mappings in Complete Metric-Like Spaces
}

\author{
Hamed H. Alsulami, ${ }^{1}$ Erdal Karapınar, ${ }^{1,2}$ and Hossein Piri ${ }^{3}$ \\ ${ }^{1}$ Nonlinear Analysis and Applied Mathematics Research Group (NAAM), King Abdulaziz University, Jeddah 21589, Saudi Arabia \\ ${ }^{2}$ Department of Mathematics, Atilim University, Incek, 06836 Ankara, Turkey \\ ${ }^{3}$ Department of Mathematics, Faculty of Basic Science, University of Bonab, P.O. Box 5551-761167, Bonab, Iran
}

Correspondence should be addressed to Erdal Karapınar; erdalkarapinar@yahoo.com

Received 29 August 2014; Revised 21 October 2014; Accepted 22 October 2014

Academic Editor: Abdul Latif

Copyright (c) 2015 Hamed H. Alsulami et al. This is an open access article distributed under the Creative Commons Attribution License, which permits unrestricted use, distribution, and reproduction in any medium, provided the original work is properly cited.

We introduce the notion of modified $F$-contractive mappings in the setting of complete metric-like spaces and we investigate the existence and uniqueness of fixed point of such mappings. The presented results unify, extend, and improve several results in the related literature.

\section{Introduction and Preliminaries}

Throughout the paper, $\mathbb{N}$ and $\mathbb{N}_{0}$ denote the set of positive integers and the set of nonnegative integers, respectively. Similarly, $\mathbb{R}, \mathbb{R}^{+}$, and $\mathbb{R}_{0}^{+}$represent the set of real, positive real, and nonnegative real numbers, respectively. In what follows, we recall the notion of partial metric which is an interesting generalization of the notion of metric.

Definition 1 (see [1]). Let $X$ be a nonempty set. A mapping $p: X \times X \rightarrow \mathbb{R}_{0}^{+}$is said to be a partial metric on $X$ if for all $x, y, z \in X$ the following conditions are satisfied:

$$
\begin{aligned}
& \left(p_{1}\right) x=y \text { if and only if } p(x, x)=p(x, y)=p(y, y) ; \\
& \left(p_{2}\right) p(x, x) \leq p(x, y) ; \\
& \left(p_{3}\right) p(x, y)=p(y, x) ; \\
& \left(p_{4}\right) p(x, z) \leq p(x, y)+p(y, z)-p(y, y) .
\end{aligned}
$$

In this case, the pair $(X, d)$ is called a partial metric space (PMS).

Notice that the function $d_{p}: X \times X \rightarrow \mathbb{R}_{0}^{+}$defined by

$$
d_{p}(x, y)=2 p(x, y)-p(x, x)-p(y, y)
$$

satisfies the conditions of a metric on $X$. Each partial metric $p$ on $X$ generates a $T_{0}$ topology $\tau_{p}$ on $X$, whose base is a family of open $p$-balls $\left\{B_{p}(x, \varepsilon): x \in X, \varepsilon>0\right\}$ where $B_{p}(x, \varepsilon)=\{y \in X: p(x, y) \leq p(x, x)+\varepsilon\}$ for all $x \in X$ and $\varepsilon>0$. Consequently, several topological concepts can be easily defined as follows.

A sequence $\left\{x_{n}\right\}$ in the PMS $(X, p)$ converges to the limit $x$ if $p(x, x)=\lim _{n \rightarrow \infty} p\left(x, x_{n}\right)$ and is said to be a Cauchy sequence if $\lim _{n, m \rightarrow \infty} p\left(x_{n}, x_{m}\right)$ exists and is finite. A PMS $(X, p)$ is called complete if every Cauchy sequence $\left\{x_{n}\right\}$ in $X$ converges with respect to $\tau_{p}$, to a point $x \in X$ such that $p(x, x)=\lim _{n, m \rightarrow \infty} p\left(x_{n}, x_{m}\right)$. For more details, see, for example, $[1-12]$ and the related references therein.

Lemma 2 (see, e.g., $[3,4])$. Let $(X, p)$ be a complete PMS. Then consider the follwing.

(A) If $p(x, y)=0$, then $x=y$.

(B) If $x \neq y$, then $p(x, y)>0$.

(C) A sequence $\left\{x_{n}\right\}$ is a Cauchy sequence in the PMS $(X, p)$ if and only if it is a Cauchy sequence in the metric space $\left(X, d_{p}\right)$. 
(D) A PMS $(X, p)$ is complete if and only if the metric space $\left(X, d_{p}\right)$ is complete. Moreover

$$
\begin{aligned}
\lim _{n \rightarrow \infty} d_{p}\left(x, x_{n}\right) & =0 \\
\Longleftrightarrow p(x, x) & =\lim _{n \rightarrow \infty} p\left(x, x_{n}\right) \\
& =\lim _{n, m \rightarrow \infty} p\left(x_{n}, x_{m}\right) .
\end{aligned}
$$

(E) Assume $x_{n} \rightarrow z$ as $n \rightarrow \infty$ in a PMS $(X, p)$ such that $p(z, z)=0$. Then $\lim _{n \rightarrow \infty} p\left(x_{n}, y\right)=p(z, y)$ for every $y \in X$.

Now, we state the definition of metric-like (dislocated) function that was first introduced by Hitzler [13] and reintroduced later by Amini-Harandi [14].

Definition 3 (see [13]). Let $X$ be a nonempty set. A mapping $\sigma: X \times X \rightarrow \mathbb{R}_{0}^{+}$is said to be metric-like (dislocated) on $X$, if for all $x, y, z \in X$ the following conditions are satisfied:

$$
\begin{aligned}
& \left(\sigma_{1}\right) \text { if } \sigma(x, y)=0 \text { then } x=y \text {; } \\
& \left(\sigma_{2}\right) \sigma(x, y)=\sigma(y, x) ; \\
& \left(\sigma_{3}\right) \sigma(x, y) \leq \sigma(x, z)+\sigma(z, y) .
\end{aligned}
$$

The pair $(X, \sigma)$ is called dislocated (metric-like) space.

Remark 4 (see [14]). Every partial metric space is a metriclike space.

A sequence $\left\{x_{n}\right\}_{n=1}^{\infty}$, in a metric-like space $(X, \sigma)$,

(a) converges to $x \in X$ if $\lim _{n \rightarrow \infty} \sigma\left(x_{n}, x\right)=\sigma(x, x)$,

(b) is called Cauchy in $(X, \sigma)$, if $\lim _{n, m \rightarrow \infty} \sigma\left(x_{n}, x_{m}\right)$ exists and is finite.

A metric-like space $(X, \sigma)$ is said to be complete if and only if every Cauchy sequence $\left\{x_{n}\right\}_{n=1}^{\infty}$ in $X$ converges to $x \in X$ so that

$$
\lim _{n, m \rightarrow \infty} \sigma\left(x_{n}, x_{m}\right)=\lim _{n \rightarrow \infty} \sigma\left(x_{n}, x\right)=\sigma(x, x) .
$$

We recall next some basic definitions and crucial results on the topic. In this paper, we follow the notations of AminiHarandi [14].

Definition 5 (see [14]). Let $(X, \sigma)$ be a metric-like space and $U$ a subset of $X$. One says that $U$ is $\sigma$-open subset of $X$, if for all $x \in X$ there exists $r>0$ such that $B_{\sigma}(x, r) \subseteq U$. Also, $V \subseteq X$ is called a $\sigma$-closed subset of $X$ if $(X \backslash V)$ is $\sigma$-open subset of $X$.

Lemma 6 (see $[15])$. Let $(X, \sigma)$ be a metric-like space. Then,

(A) if $\sigma(x, y)=0$ then $\sigma(x, x)=\sigma(y, y)=0$;

(B) if $\left\{x_{n}\right\}$ is a sequence such that $\lim _{n \rightarrow \infty} \sigma\left(x_{n}, x_{n+1}\right)=0$, then one has

$$
\lim _{n \rightarrow \infty} \sigma\left(x_{n}, x_{n}\right)=\lim _{n \rightarrow \infty} \sigma\left(x_{n+1}, x_{n+1}\right)=0
$$

(C) if $x \neq y$ then $\sigma(x, y)>0$;

(D) $\sigma(x, x) \leq(2 / n) \sum_{i=1}^{i=n} \sigma\left(x, x_{i}\right)$ holds for all $x_{i}, x \in X$ where $1 \leq i \leq n$;

(E) if $\left\{x_{n}\right\}$ is a sequence in a $\sigma$-closed subset $V$ of $X$ with $x_{n} \rightarrow x$ as $n \rightarrow \infty$, then $x \in V$;

(F) if $\left\{x_{n}\right\}$ is a sequence in $X$ such that $x_{n} \rightarrow x$ as $n \rightarrow \infty$ and $\sigma(x, x)=0$, then $\lim _{n \rightarrow \infty} \sigma\left(x_{n}, y\right)=\sigma(x, y)$ for all $y \in X$.

Definition 7. Let $(X, \sigma)$ and $(Y, \rho)$ be metric-like spaces and $\left\{x_{n}\right\}_{n=1}^{\infty}$ a sequence in $X$ such that $x_{n} \rightarrow x$. A mapping $f$ : $X \rightarrow Y$ is said to be continuous at a point $x \in X$ if $f\left(x_{n}\right) \rightarrow$ $f(x)$.

In this paper, we modify the notion of $F$-contraction that was introduced by Wardowski [16] and investigate the existence of a fixed point of such modified $F$-contractive mapping in the context of complete metric-like spaces. We consider also an example to illustrate the main result.

\section{Main Result}

In this section we present our main theorems. We start with the following definition.

Definition 8. Let $(X, \sigma)$ be a metric-like space. A selfmapping $T: X \rightarrow X$ is said to be modified $F$-contraction of type I if there exists $\tau>0$ such that

$$
\begin{aligned}
\frac{1}{2} \sigma(x, T x)< & \sigma(x, y) \Longrightarrow \tau+F(\sigma(T x, T y)) \\
\leq & \alpha F(\sigma(x, y))+\beta F(\sigma(x, T x)) \\
& +\gamma F(\sigma(y, T y))
\end{aligned}
$$

for all $x, y \in X$ with $x \neq y$ where $\gamma \in[0,1)$ and $\alpha, \beta \in[0,1]$ are real numbers such that $\alpha+\beta+\gamma=1$ and $F: \mathbb{R}^{+} \rightarrow \mathbb{R}$ is a mapping satisfying the following conditions:

$\left(F_{1}\right) F$ is strictly increasing; that is, for all $\alpha, \beta \in \mathbb{R}^{+}$such that $\alpha<\beta, F(\alpha)<F(\beta)$,

$\left(F_{2}\right)$ for any sequence $\left\{\alpha_{n}\right\}_{n=1}^{\infty}$ of positive real numbers $\lim _{n \rightarrow \infty} \alpha_{n}=0$ if and only if $\lim _{n \rightarrow \infty} F\left(\alpha_{n}\right)=-\infty$.

Theorem 9. Let $(X, \sigma)$ be a complete metric-like space and $T$ a modified F-contraction of type I. Then, $T$ has a fixed point $v \in X$; that is, $T v=v$.

Proof. For an arbitrary $x \in X$, we construct a sequence $\left\{x_{n}\right\}$ in the following way:

$$
x=x_{0}, \quad x_{n+1}=T x_{n}, \quad \forall n \in \mathbb{N}_{0} .
$$

If there exists $n_{0} \in \mathbb{N}$ such that $\sigma\left(x_{n_{0}}, x_{n_{0}+1}\right)=0$, then $v=$ $x_{n_{0}}$ is the desired fixed point of $T$ which completes the proof. Consequently, we suppose that $0<\sigma\left(x_{n}, x_{n+1}\right)$ for every $n \epsilon$ $\mathbb{N}_{0}$. Thus, we have

$$
\frac{1}{2} \sigma\left(x_{n}, T x_{n}\right)=\frac{1}{2} \sigma\left(x_{n}, x_{n+1}\right)<\sigma\left(x_{n}, x_{n+1}\right), \quad \forall n \in \mathbb{N} .
$$


By the hypothesis of the theorem, we have

$$
\begin{aligned}
\tau+F\left(\sigma\left(T x_{n}, T x_{n+1}\right)\right) \leq & \alpha F\left(\sigma\left(x_{n}, x_{n+1}\right)\right) \\
& +\beta F\left(\sigma\left(x_{n}, T x_{n}\right)\right) \\
& +\gamma F\left(\sigma\left(x_{n+1}, T x_{n+1}\right)\right),
\end{aligned}
$$

and hence

$$
\tau+(1-\gamma) F\left(\sigma\left(x_{n+1}, x_{n+2}\right)\right) \leq(\alpha+\beta) F\left(\sigma\left(x_{n}, x_{n+1}\right)\right) .
$$

Since $\alpha+\beta+\gamma=1$, we get

$$
\begin{aligned}
F\left(\sigma\left(x_{n+1}, x_{n+2}\right)\right) & \leq F\left(\sigma\left(x_{n}, x_{n+1}\right)\right)-\frac{\tau}{\alpha+\beta} \\
& <F\left(\sigma\left(x_{n}, x_{n+1}\right)\right) .
\end{aligned}
$$

So from $\left(F_{1}\right)$, we conclude that

$$
\sigma\left(x_{n+1}, x_{n+2}\right)<\sigma\left(x_{n}, x_{n+1}\right), \quad \forall n \in \mathbb{N} .
$$

Therefore, $\left\{\sigma\left(x_{n}, x_{n+1}\right)\right\}_{n=1}^{\infty}$ is a decreasing sequence of real numbers which is bounded below. This implies that $\left\{\sigma\left(x_{n}, x_{n+1}\right)\right\}_{n=1}^{\infty}$ converges and

$$
\lim _{n \rightarrow \infty} \sigma\left(x_{n}, x_{n+1}\right)=\beta=\inf \left\{\sigma\left(x_{n}, x_{n+1}\right): n \in \mathbb{N}\right\} \text {. }
$$

We will show that $\beta=0$. Suppose, on the contrary, that $\beta>0$. For every $\varepsilon>0$ there exists $m \in \mathbb{N}$, such that

$$
\sigma\left(x_{m}, T x_{m}\right)<\beta+\varepsilon .
$$

Hence from $\left(F_{1}\right)$, we get

$$
F\left(\sigma\left(x_{m}, T x_{m}\right)\right)<F(\beta+\varepsilon) .
$$

On the other hand from (7), we have

$$
\frac{1}{2} \sigma\left(x_{m}, T x_{m}\right)<\sigma\left(x_{m}, T x_{m}\right) \text {. }
$$

Due to assumption of the theorem, we obtain

$$
\begin{aligned}
\tau+F\left(\sigma\left(T x_{m}, T^{2} x_{m}\right)\right) \leq & \alpha F\left(\sigma\left(x_{m}, T x_{m}\right)\right) \\
& +\beta F\left(\sigma\left(x_{m}, T x_{m}\right)\right) \\
& +\gamma F\left(\sigma\left(T x_{m}, T^{2} x_{m}\right)\right),
\end{aligned}
$$

which is equivalent to

$$
\tau+(1-\gamma) F\left(\sigma\left(T x_{m}, T^{2} x_{m}\right)\right)<(\alpha+\beta) F\left(\sigma\left(x_{m}, T x_{m}\right)\right) .
$$

Consequently, we derive that

$$
F\left(\sigma\left(T x_{m}, T^{2} x_{m}\right)\right)<F\left(\sigma\left(x_{m}, T x_{m}\right)\right)-\frac{\tau}{\alpha+\beta},
$$

since $\alpha+\beta+\gamma=1$. On account of (7), we have $(1 / 2) \sigma\left(T x_{m}\right.$, $\left.T^{2} x_{m}\right)<\sigma\left(T x_{m}, T^{2} x_{m}\right)$; thus by assumption of the theorem, we have

$$
\begin{aligned}
\tau+F\left(\sigma\left(T^{2} x_{m}, T^{3} x_{m}\right)\right) \leq & \alpha F\left(\sigma\left(T x_{m}, T^{2} x_{m}\right)\right) \\
& +\beta F\left(\sigma\left(T x_{m}, T^{2} x_{m}\right)\right) \\
& +\gamma F\left(\sigma\left(T^{2} x_{m}, T^{3} x_{m}\right)\right),
\end{aligned}
$$

which yields

$$
\begin{aligned}
\tau+ & (1-\gamma) F\left(\sigma\left(T^{2} x_{m}, T^{3} x_{m}\right)\right) \\
& \leq(\alpha+\beta) F\left(\sigma\left(T x_{m}, T^{2} x_{m}\right)\right) .
\end{aligned}
$$

Owing to the fact that $\alpha+\beta+\gamma=1$, we obtain that

$$
F\left(\sigma\left(T^{2} x_{m}, T^{3} x_{m}\right)\right) \leq F\left(\sigma\left(T x_{m}, T^{2} x_{m}\right)\right)-\frac{\tau}{\alpha+\beta} .
$$

Now by using (14) and continuing in the same way as in the derivation of (18) and (21), we deduce

$$
\begin{aligned}
F\left(\sigma\left(T^{n} x_{m}, T^{n+1} x_{m}\right)\right) & \leq F\left(\sigma\left(T^{n} x_{m}, T^{n-1} x_{m}\right)\right)-\frac{\tau}{\alpha+\beta} \\
& \leq F\left(\sigma\left(T^{n-1} x_{m}, T^{n-2} x_{m}\right)\right)-\frac{2 \tau}{\alpha+\beta} \\
& \vdots \\
& \leq F\left(\sigma\left(T x_{m}, x_{m}\right)\right)-\frac{n \tau}{\alpha+\beta} \\
& <F(\beta+\varepsilon)-\frac{n \tau}{\alpha+\beta} .
\end{aligned}
$$

This implies that $\lim _{n \rightarrow \infty} F\left(\sigma\left(T^{n} x_{m}, T^{n+1} x_{m}\right)\right)=-\infty$. Regarding $\left(F_{2}\right)$, we have $\lim _{n \rightarrow \infty} \sigma\left(T^{n} x_{m}, T^{n+1} x_{m}\right)=0$, and thus, there exists $N_{1} \in \mathbb{N}$ such that $\sigma\left(T^{n} x_{m}, T^{n+1} x_{m}\right)<\beta$, $\forall n \geq N_{1}$. Therefore, from (6), we get

$$
\sigma\left(x_{m+n}, T x_{m+n}\right)<\beta, \quad \forall n \geq N_{1} .
$$

This contradicts the definition of $\beta$ given in (12). Then we get $\beta=0$ and from (12) we conclude

$$
\lim _{n \rightarrow \infty} \sigma\left(x_{n}, x_{n+1}\right)=0 .
$$

In the next step, we claim that

$$
\lim _{n, m \rightarrow \infty} \sigma\left(x_{n}, x_{m}\right)=0
$$

Suppose, on the contrary, that there exist $\varepsilon>0$ and sequences $\{p(n)\}_{n=1}^{\infty}$ and $\{q(n)\}_{n=1}^{\infty}$ of natural numbers such that

$$
\begin{array}{r}
p(n)>q(n)>n, \\
\sigma\left(x_{p(n)}, x_{q(n)}\right) \geq \varepsilon, \\
\sigma\left(x_{p(n)-1}, x_{q(n)}\right)<\varepsilon,
\end{array}
$$


By triangular inequality, we have

$$
\begin{aligned}
\varepsilon & \leq \sigma\left(x_{p(n)}, x_{q(n)}\right) \\
& \leq \sigma\left(x_{p(n)}, x_{p(n)-1}\right)+\sigma\left(x_{p(n)-1}, x_{q(n)}\right) \\
& \leq \sigma\left(x_{p(n)}, x_{p(n)-1}\right)+\varepsilon \\
& =\sigma\left(x_{p(n)-1}, T x_{p(n)-1}\right)+\varepsilon, \quad \forall n \in \mathbb{N} .
\end{aligned}
$$

It follows from (24), (27), and Squeezing Theorem that

$$
\lim _{n \rightarrow \infty} \sigma\left(x_{p(n)}, x_{q(n)}\right)=\varepsilon .
$$

From (24), (26), and (28), there exists $N_{2} \in \mathbb{N}$ such that

$$
\begin{array}{r}
\frac{1}{2} \sigma\left(x_{p(n)}, T x_{p(n)}\right)<\frac{1}{2} \varepsilon<\varepsilon \leq \sigma\left(x_{p(n)}, x_{q(n)}\right)<2 \varepsilon, \\
\forall n>N_{2} .
\end{array}
$$

Hence from (29), $\left(F_{1}\right)$, and the hypothesis of the theorem, we have

$$
\begin{aligned}
\tau+ & F\left(\sigma\left(T x_{p(n)}, T x_{q(n)}\right)\right) \\
\leq & \alpha F\left(\sigma\left(x_{p(n)}, x_{q(n)}\right)\right)+\beta F\left(\sigma\left(x_{p(n)}, T x_{p(n)}\right)\right) \\
& +\gamma F\left(\sigma\left(x_{q(n)}, T x_{q(n)}\right)\right) \\
< & \alpha F(2 s \varepsilon)+\beta F\left(\sigma\left(x_{p(n)}, T x_{p(n)}\right)\right) \\
& +\gamma F\left(\sigma\left(x_{q(n)}, T x_{q(n)}\right)\right), \quad \forall n>N_{2} .
\end{aligned}
$$

From $(24)$ and $\left(F_{2}\right)$ it follows that

$$
\lim _{n \rightarrow \infty} F\left(\sigma\left(T x_{p(n)}, T x_{q(n)}\right)\right)=-\infty
$$

and hence we get

$\lim _{n \rightarrow \infty} \sigma\left(T x_{p(n)}, T x_{q(n)}\right)=0 \Longleftrightarrow \lim _{n \rightarrow \infty} \sigma\left(x_{p(n)+1}, x_{q(n)+1}\right)=0$.

However, this contradicts the relation (26). Hence $\lim _{m, n \rightarrow \infty} \sigma\left(x_{n}, x_{m}\right)=0$. Therefore $\left\{x_{n}\right\}_{n=1}^{\infty}$ is a Cauchy sequence in $X$. By the completeness of $(X, \sigma)$ there exists $v \in X$ such that

$$
\sigma(v, v)=\lim _{n \rightarrow \infty} \sigma\left(x_{n}, v\right)=\lim _{n, m \rightarrow \infty} \sigma\left(x_{n}, x_{m}\right)=0 .
$$

Next, we will prove that, for every $n \in \mathbb{N}$,

$$
\begin{aligned}
& \frac{1}{2} \sigma\left(x_{n}, T x_{n}\right)<\sigma\left(x_{n}, v\right) \quad \text { or } \\
& \frac{1}{2} \sigma\left(T x_{n}, T^{2} x_{n}\right)<\sigma\left(T x_{n}, v\right),
\end{aligned}
$$

Arguing by contradiction, we assume that there exists $m \in \mathbb{N}$ such that

$$
\begin{aligned}
\frac{1}{2} \sigma\left(x_{m}, T x_{m}\right) & \geq \sigma\left(x_{m}, v\right), \\
\frac{1}{2} \sigma\left(T x_{m}, T^{2} x_{m}\right) & \geq \sigma\left(T x_{m}, v\right) .
\end{aligned}
$$

From (18) and $\left(F_{1}\right)$, we have

$$
\sigma\left(T x_{m}, T^{2} x_{m}\right)<\sigma\left(x_{m}, T x_{m}\right)
$$

It follows from (35) and (36) that

$$
\begin{aligned}
\left(x_{m}, T x_{m}\right) & \leq \sigma\left(x_{m}, v\right)+\sigma\left(v, T x_{m}\right) \\
& \leq \frac{1}{2} \sigma\left(x_{m}, T x_{m}\right)+\frac{1}{2} \sigma\left(T x_{m}, T^{2} x_{m}\right) \\
& <\frac{1}{2} \sigma\left(x_{m}, T x_{m}\right)+\frac{1}{2} \sigma\left(x_{m}, T x_{m}\right) \\
& =\sigma\left(x_{m}, T x_{m}\right) .
\end{aligned}
$$

Obviously, this is a contradiction. Hence, inequality (34) is satisfied. Regarding the assumption of the theorem, (34) implies that either

$$
\begin{aligned}
\tau+F\left(\sigma\left(T x_{n}, T v\right)\right) \leq & \alpha F\left(\sigma\left(x_{n}, v\right)\right) \\
& +\beta F\left(\sigma\left(x_{n}, T x_{n}\right)\right)+\gamma F(\sigma(v, T v))
\end{aligned}
$$

or

$$
\begin{aligned}
\tau+F\left(\sigma\left(T^{2} x_{n}, T v\right)\right) \leq & \alpha F\left(\sigma\left(T x_{n}, v\right)\right) \\
& +\beta F\left(\sigma\left(T x_{n}, T^{2} x_{n}\right)\right) \\
& +\gamma F(\sigma(v, T v)),
\end{aligned}
$$

for every $n \in \mathbb{N}$. In the first case, because of $\left(F_{2}\right)$, the limits in (24) and (33) imply

$$
\begin{gathered}
\lim _{n \rightarrow \infty} F\left(\sigma\left(x_{n}, v\right)\right)=-\infty, \\
\lim _{n \rightarrow \infty} F\left(\sigma\left(x_{n}, T x_{n}\right)\right)=-\infty .
\end{gathered}
$$

Thus, letting $n \rightarrow \infty$ in (38), we conclude that

$$
\lim _{n \rightarrow \infty} F\left(\sigma\left(T x_{n}, T v\right)\right)=-\infty
$$

Again by $\left(F_{2}\right)$, we observe that

$$
\lim _{n \rightarrow \infty} \sigma\left(T x_{n}, T v\right)=0
$$

On the other hand, from (6), we have

$$
\begin{aligned}
\sigma(v, T v) & \leq \sigma\left(v, T x_{n}\right)+\sigma\left(T x_{n}, T v\right) \\
& =\sigma\left(v, x_{n+1}\right)+\sigma\left(T x_{n}, T v\right) .
\end{aligned}
$$


It follows from (33) and (42) that $\sigma(v, T v)=0$; therefore $v=$ Tv.

In the second case from (6), we have

$$
\begin{aligned}
F\left(\sigma\left(T^{2} x_{n}, T v\right)\right)< & \tau+F\left(\sigma\left(T^{2} x_{n}, T v\right)\right) \\
\leq & \alpha F\left(\sigma\left(T x_{n}, v\right)\right)+\beta F\left(\sigma\left(T x_{n}, T^{2} x_{n}\right)\right) \\
& +\gamma F(\sigma(v, T v)) \\
= & \alpha F\left(\sigma\left(x_{n+1}, v\right)\right)+\beta F\left(\sigma\left(x_{n+1}, T x_{n+1}\right)\right) \\
& +\gamma F(D(v, T v)) .
\end{aligned}
$$

Then employing (24), (33), and $\left(F_{2}\right)$, we conclude that $\lim _{n \rightarrow \infty} F\left(\sigma\left(T^{2} x_{n}, T v\right)\right)=-\infty$. Equivalently, from $\left(F_{2}\right)$ we get

$$
\lim _{n \rightarrow \infty} \sigma\left(T^{2} x_{n}, T v\right)=0
$$

Using (6), we obtain

$$
\begin{aligned}
\sigma(v, T v) & \leq \sigma\left(v, T^{2} x_{n}\right)+\sigma\left(T^{2} x_{n}, T v\right) \\
& =\sigma\left(v, x_{n+2}\right)+\sigma\left(T^{2} x_{n}, T v\right) .
\end{aligned}
$$

Finally, from (33) and (45) it follows that $\sigma(v, T v)=0$; therefore $v=T v$. Hence, $v$ is a fixed point of $T$.

Definition 10. Let $(X, \sigma)$ be a metric-like space. A mapping $T: X \rightarrow X$ is said to be a modified $F$-contraction of type II if there exists $\tau>0$ such that

$$
\frac{1}{2} \sigma(x, T x)<\sigma(x, y) \Longrightarrow \tau+F(\sigma(T x, T y)) \leq F(\sigma(x, y)),
$$

for all $x, y \in X$ with $x \neq y$ where $F: \mathbb{R}^{+} \rightarrow \mathbb{R}$ is a mapping satisfying the conditions $\left(F_{1}\right)$ and $\left(F_{2}\right)$ stated in Definition 8 .

Theorem 11. Let $(X, \sigma)$ be a complete metric-like space and $T$ a modified F-contraction of type II. Then, $T$ has a fixed point $v \in X$; that is, $T v=v$.

Proof. It is sufficient to take $\alpha=1$ and $\beta=\gamma=0$ in Theorem 9.

Definition 12. Let $(X, p)$ be a partial metric space. A selfmapping $T: X \rightarrow X$ is said to be a modified $F$-contraction of type I if there exists $\tau>0$ such that

$$
\begin{aligned}
\frac{1}{2} p(x, T x)<p(x, y) & \\
\Longrightarrow \tau+F(p(T x, T y)) \leq & \alpha F(p(x, y))+\beta F(p(x, T x)) \\
& +\gamma F(p(y, T y)),
\end{aligned}
$$

for all $x, y \in X$ with $x \neq y$ where $\gamma \in[0,1)$ and $\alpha, \beta \in[0,1]$ are real numbers such that $\alpha+\beta+\gamma=1$ and $F: \mathbb{R}^{+} \rightarrow \mathbb{R}$ is a mapping satisfying the following conditions:
$\left(F_{1}\right) F$ is strictly increasing; that is, for all $\alpha, \beta \in \mathbb{R}^{+}$such that $\alpha<\beta, F(\alpha)<F(\beta)$,

$\left(F_{2}\right)$ for any sequence $\left\{\alpha_{n}\right\}_{n=1}^{\infty}$ of positive real numbers $\lim _{n \rightarrow \infty} \alpha_{n}=0$ if and only if $\lim _{n \rightarrow \infty} F\left(\alpha_{n}\right)=-\infty$.

Theorem 13. Let $(X, p)$ be a complete partial metric space and $T$ a modified $F$-contraction of type I. Then, $T$ has a unique fixed point $v \in X$; that is, $T v=v$.

Proof. Since every partial metric space is metric-like space (see, e.g., Remark 4), the existence of a fixed point $v \in X$ of the mapping $T$ is guaranteed by Theorem 9 . Thus, it is sufficient to show that $v$ is the unique fixed point of $T$. Suppose, on the contrary, that $w \in X$ is another fixed point of $T$ such that $v \neq w$. Then, we have $p(v, w)>0$. If $p(v, v)=0$, we have

$$
0=\frac{1}{2} p(v, v)=\frac{1}{2} p(v, T v)<p(v, w) .
$$

If $p(v, v)>0$, then the inequality

$$
\frac{1}{2} p(v, T v)=\frac{1}{2} p(v, v)<p(v, v) \leq p(v, w)
$$

follows from the condition $\left(p_{2}\right)$ in Definition 1 . In any case, the left-hand side of (48) is fulfilled. Hence, we have

$$
\begin{aligned}
\tau+F(p(T v, T w)) \leq & \alpha F(p(v, w))+\beta F(p(v, T v)) \\
& +\gamma F(p(w, T w)) \\
= & \alpha F(p(v, w))+\beta F(p(v, v)) \\
& +\gamma F(p(w, w)) .
\end{aligned}
$$

On the other hand, from $\left(p_{2}\right)$, we have $p(v, v) \leq p(v, w)$ and $p(w, w) \leq p(v, w)$. Regarding $\left(F_{2}\right)$, we get that

$$
\begin{gathered}
F(p(v, v)) \leq F(p(v, w)), \\
F(p(w, w)) \leq F(p(v, w)) .
\end{gathered}
$$

Combining (51) and (52), we conclude that

$$
\begin{aligned}
F(p(v, w))< & \tau+F(p(v, w))=\tau+F(p(T v, T w)) \\
< & \alpha F(p(v, w))+\beta F(p(v, w)) \\
& +\gamma F(p(v, w)) \\
= & (\alpha+\beta+\gamma) F(p(v, w)) \\
= & F(p(v, w)),
\end{aligned}
$$

since $\tau>0$ and $\alpha+\beta+\gamma=1$. This is a contradiction and hence $v=w$.

Analogously, we conclude a result similar to Theorem 11 by introducing the next definition.

Definition 14. Let $(X, p)$ be a partial space. A mapping $T$ : $X \rightarrow X$ is said to be a modified $F$-contraction of type II if there exists $\tau>0$ such that

$$
\frac{1}{2} p(x, T x)<p(x, y) \Longrightarrow \tau+F(p(T x, T y)) \leq F(p(x, y)) \text {, }
$$


for all $x, y \in X$ with $x \neq y$ where $F: \mathbb{R}^{+} \rightarrow \mathbb{R}$ is a mapping satisfying the conditions $\left(F_{1}\right)$ and $\left(F_{2}\right)$ stated in Definition 12 .

Theorem 15. Let $(X, p)$ be a complete partial space and $T$ a modified F-contraction of type II. Then, $T$ has a unique fixed point $v \in X$; that is, $T v=v$.

Proof. It is sufficient to take $\alpha=1$ and $\beta=\gamma=0$ in Theorem 13.

Definition 16. Let $(X, \sigma)$ be a metric-like space. A selfmapping $T: X \rightarrow X$ is said to be modified $F$-contraction of type III if there exists $\tau>0$ such that

$$
\begin{aligned}
& \sigma(T x, T y)>0 \\
& \Rightarrow \tau+F(\sigma(T x, T y))< \\
& =\alpha F(\sigma(x, y))+\beta F(\sigma(x, T x)) \\
& +\gamma F(\sigma(y, T y)),
\end{aligned}
$$

for all $x, y \in X$ with $x \neq y$ where $\gamma \in[0,1)$ and $\alpha, \beta \in[0,1]$ are real numbers such that $\alpha+\beta+\gamma=1$ and $F: \mathbb{R}^{+} \rightarrow \mathbb{R}$ is a mapping satisfying the conditions $\left(F_{1}\right)$ and $\left(F_{2}\right)$ introduced in Definition 8.

Theorem 17. Let $(X, \sigma)$ be a complete metric-like space and $T$ a continuous modified $F$-contraction of type III. If $\sigma(T x, T x) \leq$ $\sigma(x, x)$ for all $x \in X$, then $T$ has a fixed point $v \in X$; that is, $T v=v$.

Proof. As in the proof of Theorem 9, we construct an iterative sequence $\left\{x_{n}\right\}$ in the following way. Take and arbitrary $x \in X$ and set $x=x_{0}$ and

$$
x_{n+1}=T x_{n}, \quad \forall n \in \mathbb{N}_{0} .
$$

Notice that if $\sigma\left(x_{n_{0}}, x_{n_{0}+1}\right)=0$ for some $n_{0} \in \mathbb{N}_{0}$, the proof is completed. Suppose that

$$
\sigma\left(x_{n-1}, x_{n}\right)>0
$$

for all $n \in \mathbb{N}$. Thus, (55) yields that

$$
\begin{aligned}
& \tau+F\left(\sigma\left(x_{n}, T x_{n+1}\right)\right) \\
&= \tau+F\left(\sigma\left(T x_{n-1}, T x_{n}\right)\right) \\
& \leq \alpha F\left(\sigma\left(x_{n-1}, x_{n}\right)\right)+\beta F\left(\sigma\left(x_{n-1}, T x_{n-1}\right)\right) \\
&+\gamma F\left(\sigma\left(x_{n}, T x_{n}\right)\right) \\
&= \alpha F\left(\sigma\left(x_{n-1}, x_{n}\right)\right)+\beta F\left(\sigma\left(x_{n-1}, x_{n}\right)\right) \\
&+\gamma F\left(\sigma\left(x_{n}, x_{n+1}\right)\right),
\end{aligned}
$$

which can be written as

$$
\tau+(1-\gamma) F\left(\sigma\left(x_{n}, x_{n+1}\right)\right) \leq(\alpha+\beta) F\left(\sigma\left(x_{n-1}, x_{n}\right)\right) .
$$

Regarding the assumption $\alpha+\beta+\gamma=1$, we get

$$
\begin{aligned}
F\left(\sigma\left(x_{n}, x_{n+1}\right)\right) & \leq F\left(\sigma\left(x_{n-1}, x_{n}\right)\right)-\frac{\tau}{\alpha+\beta} \\
& <F\left(\sigma\left(x_{n-1}, x_{n}\right)\right) .
\end{aligned}
$$

From $\left(F_{1}\right)$, we conclude that

$$
\sigma\left(x_{n}, x_{n+1}\right)<\sigma\left(x_{n-1}, x_{n}\right), \quad \forall n \in \mathbb{N}
$$

Therefore, $\left\{\sigma\left(x_{n}, x_{n+1}\right)\right\}_{n=1}^{\infty}$ is a decreasing sequence of real numbers which is bounded from below. Hence, it converges and

$$
\lim _{n \rightarrow \infty} \sigma\left(x_{n}, x_{n+1}\right)=\beta=\inf \left\{\sigma\left(x_{n}, x_{n+1}\right): n \in \mathbb{N}\right\}
$$

We will show that $\beta=0$ by method of Reductio ad absurdum. Suppose that $\beta>0$. Thus, for every $\varepsilon>0$ there exists $m \in \mathbb{N}$, such that $\sigma\left(x_{m}, x_{m+1}\right)<\beta+\varepsilon$. Because of $\left(F_{1}\right)$, we have

$$
F\left(\sigma\left(x_{m}, T x_{m}\right)\right)<F(\beta+\varepsilon) \text {. }
$$

On the other hand, it follows from (57) that $0<\sigma\left(x_{m}\right.$, $\left.x_{m+1}\right)=\sigma\left(T x_{m-1}, T x_{m}\right)$, which implies

$$
\begin{aligned}
& \tau+F\left(\sigma\left(T x_{m-1}, T x_{m}\right)\right) \\
& \leq \alpha F\left(\sigma\left(x_{m-1}, x_{m}\right)\right)+\beta F\left(\sigma\left(x_{m-1}, T x_{m-1}\right)\right) \\
&+\gamma F\left(\sigma\left(x_{m}, T x_{m}\right)\right) \\
&= \alpha F\left(\sigma\left(x_{m-1}, x_{m}\right)\right)+\beta F\left(\sigma\left(x_{m-1}, x_{m}\right)\right) \\
&+\gamma F\left(\sigma\left(T x_{m-1}, T x_{m}\right)\right)
\end{aligned}
$$

due to (55). Consequently, we have

$$
\tau+(1-\gamma) F\left(\sigma\left(T x_{m-1}, T x_{m}\right)\right)<(\alpha+\beta) F\left(\sigma\left(x_{m-1}, x_{m}\right)\right) .
$$

Since $\alpha+\beta+\gamma=1$, we obtain that

$$
F\left(\sigma\left(T x_{m-1}, T x_{m}\right)\right) \leq F\left(\sigma\left(x_{m-1}, x_{m}\right)\right)-\frac{\tau}{\alpha+\beta}
$$

Again from (57), we have $0<\sigma\left(T x_{m}, T x_{m+1}\right)$; thus, by the hypothesis of the theorem

$$
\begin{aligned}
& \tau+F\left(\sigma\left(T x_{m}, T x_{m+1}\right)\right) \\
& \leq \alpha F\left(\sigma\left(x_{m}, x_{m+1}\right)\right)+\beta F\left(\sigma\left(x_{m}, T x_{m}\right)\right) \\
&+\gamma F\left(\sigma\left(x_{m+1}, T x_{m+1}\right)\right) \\
&= \alpha F\left(\sigma\left(x_{m}, x_{m+1}\right)\right)+\beta F\left(\sigma\left(x_{m}, x_{m+1}\right)\right) \\
&+\gamma F\left(\sigma\left(T x_{m}, T x_{m+1}\right)\right),
\end{aligned}
$$

which results in $\tau+(1-\gamma) F\left(\sigma\left(T x_{m}, T x_{m+1}\right)\right) \leq(\alpha+$ $\beta) F\left(\sigma\left(x_{m}, x_{m+1}\right)\right)$. Taking into account that $\alpha+\beta+\gamma=1$, we derive

$$
F\left(\sigma\left(T x_{m}, T x_{m+1}\right)\right) \leq F\left(\sigma\left(x_{m}, x_{m+1}\right)\right)-\frac{\tau}{\alpha+\beta} .
$$


Now we employ (63) and applying a procedure similar to that used in derivation of (66) and (68), we obtain

$$
\begin{aligned}
& F\left(\sigma\left(x_{m+n}, x_{m+n+1}\right)\right) \\
& =F\left(\sigma\left(T x_{m+n-1}, T x_{m+n}\right)\right) \\
& \leq F\left(\sigma\left(x_{m+n-1}, x_{m+n}\right)\right)-\frac{\tau}{\alpha+\beta} \\
& =F\left(\sigma\left(T x_{m+n-2}, T x_{m+n-1}\right)\right)-\frac{\tau}{\alpha+\beta} \\
& \leq F\left(\sigma\left(x_{m+n-2}, x_{m+n-1}\right)\right)-\frac{2 \tau}{\alpha+\beta} \\
& =F\left(\sigma\left(T x_{m+n-3}, T x_{m+n-2}\right)\right)-\frac{2 \tau}{\alpha+\beta} \\
& \leq F\left(\sigma\left(x_{m+n-3}, x_{m+n-2}\right)\right)-\frac{3 \tau}{\alpha+\beta} \\
& \leq F\left(\sigma\left(x_{m+1}, x_{m+2}\right)\right)-\frac{(n-1) \tau}{\alpha+\beta} \\
& =F\left(\sigma\left(T x_{m}, T x_{m+1}\right)\right)-\frac{(n-1) \tau}{\alpha+\beta} \\
& \leq F(\beta+\varepsilon)-\frac{n \tau}{\alpha+\beta} .
\end{aligned}
$$

This implies that

$$
\lim _{n \rightarrow \infty} F\left(\sigma\left(x_{m+n}, x_{m+n+1}\right)\right)=-\infty
$$

Then, from $\left(F_{2}\right)$ we have $\lim _{n \rightarrow \infty} \sigma\left(x_{m+n}, x_{m+n+1}\right)=0$, so that there exists $N_{1} \in \mathbb{N}$ such that

$$
\sigma\left(x_{m+n}, x_{m+n+1}\right)<\beta, \quad \forall n \geq N_{1} .
$$

However, this contradicts the definition of $\beta$ given in (62). Thus, $\beta=0$ and from (62) we conclude

$$
\lim _{n \rightarrow \infty} \sigma\left(x_{n}, x_{n+1}\right)=0
$$

In the sequel, we will show that $\lim _{n, m \rightarrow \infty} \sigma\left(x_{n}, x_{m}\right)=$ 0 . Assume the contrary; that is, let there exist $\varepsilon>0$ and sequences $\{p(n)\}_{n=1}^{\infty}$ and $\{q(n)\}_{n=1}^{\infty}$ of natural numbers such that

$$
\begin{aligned}
& p(n)>q(n)>n, \\
& \sigma\left(x_{p(n)}, x_{q(n)}\right) \geq \varepsilon, \\
& \sigma\left(x_{p(n)-1}, x_{q(n)}\right)<\varepsilon, \\
& \forall n \in \mathbb{N} .
\end{aligned}
$$

Observe that by the triangle inequality we have

$$
\begin{aligned}
\varepsilon & \leq \sigma\left(x_{p(n)}, x_{q(n)}\right) \\
& \leq \sigma\left(x_{p(n)}, x_{p(n)-1}\right)+\sigma\left(x_{p(n)-1}, x_{q(n)}\right) \\
& \leq \sigma\left(x_{p(n)}, x_{p(n)-1}\right)+\varepsilon \\
& =\sigma\left(x_{p(n)-1}, T x_{p(n)-1}\right)+\varepsilon, \quad \forall n \in \mathbb{N} .
\end{aligned}
$$

It follows from (72), (74), and the Squeeze Theorem that

$$
\lim _{n \rightarrow \infty} \sigma\left(x_{p(n)}, x_{q(n)}\right)=\varepsilon .
$$

Therefore, there exists $N_{2} \in \mathbb{N}$ such that

$$
\varepsilon \leq \sigma\left(x_{p(n)}, x_{q(n)}\right)<2 \varepsilon \quad \forall n>N_{2} .
$$

Hence from (76), $\left(F_{1}\right)$, and assumption of theorem, we have

$$
\begin{aligned}
\tau+F & \left(\sigma\left(T x_{p(n)-1}, T x_{q(n)-1}\right)\right) \\
\leq & \alpha F\left(\sigma\left(x_{p(n)-1}, x_{q(n)-1}\right)\right) \\
& +\beta F\left(\sigma\left(x_{p(n)-1}, T x_{p(n)-1}\right)\right) \\
& +\gamma F\left(\sigma\left(x_{q(n)-1}, T x_{q(n)-1}\right)\right) \\
< & \alpha F(2 s \varepsilon)+\beta F\left(\sigma\left(x_{p(n)-1}, T x_{p(n)-1}\right)\right) \\
& +\gamma F\left(\sigma\left(x_{q(n)-1}, T x_{q(n)-1}\right)\right), \quad \forall n>N_{2} .
\end{aligned}
$$

Now, using (72), (75), and $\left(F_{2}\right)$, we obtain

$$
\lim _{n \rightarrow \infty} F\left(\sigma\left(T x_{p(n)-1}, T x_{q(n)-1}\right)\right)=-\infty .
$$

However, due to $\left(F_{2}\right)$ it follows that

$$
\lim _{n \rightarrow \infty} \sigma\left(T x_{p(n)-1}, T x_{q(n)-1}\right)=0 \Longleftrightarrow \lim _{n \rightarrow \infty} \sigma\left(x_{p(n)}, x_{q(n)}\right)=0 .
$$

This is a contradiction with the relation (73). Hence $\lim _{m, n \rightarrow \infty} \sigma\left(x_{n}, x_{m}\right)=0$. By the completeness of $(X, \sigma)$ there exists $v \in X$ such that

$$
\sigma(v, v)=\lim _{n \rightarrow \infty} \sigma\left(x_{n}, v\right)=\lim _{n \rightarrow \infty} \sigma\left(x_{n}, x_{m}\right)=0 .
$$

Since $x_{n} \rightarrow v$ and $T$ is continuous, we deduce $T x_{n} \rightarrow T v$ by Definition 7. Consequently, we have

$$
\sigma(T v, T v)=\lim _{n \rightarrow \infty} \sigma\left(T x_{n}, T v\right)=\lim _{n \rightarrow \infty} \sigma\left(x_{n+1}, T v\right)
$$

According to the assumption of the theorem $\sigma(T v, T v) \leq$ $\sigma(v, v)$. Then, from (80) and (81) it follows that

$$
\lim _{n \rightarrow \infty} \sigma\left(x_{n}, v\right)=\lim _{n \rightarrow \infty} \sigma\left(x_{n}, T v\right)=0 .
$$

Since $\sigma(v, T v) \leq \sigma\left(v, x_{n}\right)+\sigma\left(x_{n}, T v\right)$, we obtain $\sigma(v, T v)=0$ and thus the condition $\left(\sigma_{1}\right)$ gives $v=T v$, which completes the proof. 
In the following result, we proved that Theorem 17 is valid in the context of partial metric space.

Theorem 18. Let $(X, p)$ be complete partial metric space and let $T$ be a continuous self-mapping on $X$ such that, for all $x \in X$, $p(T x, T x) \leq p(x, x)$. Let $\gamma \in[0,1)$ and $\alpha, \beta \in[0,1]$ be real numbers such that $\alpha+\beta+\gamma=1$. Assume that there exists $\tau>0$ such that, for all $x, y \in X$,

$$
\begin{aligned}
p(T x, T y)>0 & \\
\Longrightarrow \tau+F(p(T x, T y)) \leq & \alpha F(p(x, y))+\beta F(p(x, T x)) \\
& +\gamma F(p(y, T y))
\end{aligned}
$$

where $F: \mathbb{R}^{+} \rightarrow \mathbb{R}$ satisfies the conditions $\left(F_{1}\right)$ and $\left(F_{2}\right)$. Then, $T$ has a unique fixed point $v \in X$; that is, $T v=v$.

Proof. Since every partial metric space is a metric-like space, Theorem 17 provides the existence of a fixed point; that is, $T$ has a fixed point $v \in X$. Therefore, it is sufficient to show the uniqueness of the fixed point of $T$. Indeed, if there is another fixed point $w \in X$ of $T$, such that $v \neq w$, due to $\left(p_{1}\right)$, we have $p(v, w)>0$ and equivalently $p(T v, T w)>0$. By the assumption of theorem, we have

$$
\begin{aligned}
\tau+F(p(T v, T w))< & \alpha F(p(v, w))+\beta F(p(v, T v)) \\
& +\gamma F(p(w, T w)) \\
= & \alpha F(p(v, w))+\beta F(p(v, v)) \\
& +\gamma F(p(w, w)) .
\end{aligned}
$$

On the other hand, $\left(p_{2}\right)$ implies

$$
p(v, v) \leq p(v, w), \quad p(w, w) \leq p(v, w) .
$$

Moreover, from $\left(F_{2}\right)$, we get

$$
\begin{aligned}
& F(p(v, v)) \leq F(p(v, w)), \\
& F(p(w, w)) \leq F(p(v, w)) .
\end{aligned}
$$

Due to the fact that $\tau>0$ and $\alpha+\beta+\gamma=1$, from (84) and (86), we conclude

$$
\begin{aligned}
F(p(v, w)) & =F(p(T v, T w)) \\
& <\alpha F(p(v, w))+\beta F(p(v, w))+\gamma F(p(v, w)) \\
& =(\alpha+\beta+\gamma) F(p(v, w)) \\
& =F(p(v, w)) .
\end{aligned}
$$

However, this is a contradiction, and hence, $v=w$.

Definition 19. Let $(X, \sigma)$ be metric-like spaces. A selfmapping $T: X \rightarrow X$ is said to be modified $F$-contraction of type IV if there exists $\tau>0$ such that

$$
\sigma(T x, T y)>0 \Longrightarrow \tau+F(\sigma(T x, T y))<F(\sigma(x, y))
$$

for all $x, y \in X$ with $x \neq y$ where $F: \mathbb{R}^{+} \rightarrow \mathbb{R}$ is a mapping satisfying the conditions $\left(F_{1}\right)$ and $\left(F_{2}\right)$ introduced in Definition 8.

Theorem 20. Let $(X, \sigma)$ be a complete metric-like space and $T$ a continuous modified $F$-contraction of type IV. If $\sigma(T x, T x) \leq$ $\sigma(x, x)$ for all $x \in X$, then $T$ has a fixed point $v \in X$; that is, $T v=v$.

Proof. The proof is obvious by taking $\alpha=1$ and $\beta=\gamma=0$ in Theorem 17.

Theorem 21. Let $(X, p)$ be complete PMS and let $T$ be a continuous self-mapping on $X$ such that, for all $x \in X$, $p(T x, T x) \leq p(x, x)$. Assume that there exists $\tau>0$ such that, for all $x, y \in X$,

$$
0<p(T x, T y) \Longrightarrow \tau+F(p(T x, T y)) \leq F(p(x, y)),
$$

where $F: \mathbb{R}^{+} \rightarrow \mathbb{R}$ satisfies conditions $\left(F_{1}\right)$ and $\left(F_{2}\right)$. Then, $T$ has a unique fixed point $v \in X$; that is, $T v=v$.

Proof. The proof is trivial by taking $\alpha=1$ and $\beta=\gamma=0$ in Theorem 18.

Last, we provide an example which illustrates our results.

Example 22. Let $k$ be a real number such that $k>1, N \in \mathbb{N}$, and

$$
\begin{aligned}
& S_{0}=0, \\
& S_{1}=1, \\
& S_{2}=1+2,
\end{aligned}
$$

$$
S_{N}=1+2+\cdots+N
$$

Let $X=\left\{S_{0}, S_{1}, S_{2}, \ldots, S_{N}\right\}$ and $p$ be defined as

$$
\begin{gathered}
p: X \times X \longrightarrow \mathbb{R}_{0}^{+} \\
p(x, y)=\max \{x, y\}^{k}+|x-y| .
\end{gathered}
$$

Define a mapping $T: X \rightarrow X$ as follows:

$$
T\left(S_{0}\right)=S_{0}, \quad T\left(S_{n}\right)=S_{n-1}, \quad \forall n \in\{1,2, \ldots, N\} .
$$

Then $T$ satisfies in the conditions of Theorem 21.

Observe that $(X, p)$ is a complete partial metric space, but not a metric space. Define the function $F$ in Theorem 21 as $F(\alpha)=\ln \alpha+\alpha$. Then we get

$$
\begin{aligned}
\tau+F & (p(T x, T y)) \leq F(p(x, y)) \\
& \Longleftrightarrow \frac{p(T x, T y)}{p(x, y)} e^{p(T x, T y)-p(x, y)} \leq e^{-\tau} .
\end{aligned}
$$


Note also that, for all $0 \leq n<m \leq N$, we have

$$
\begin{aligned}
\frac{p\left(T S_{n}, T S_{m}\right)}{p\left(S_{n}, S_{m}\right)} e^{\left[p\left(T S_{n}, T S_{m}\right)-p\left(S_{n}, S_{m}\right)\right]} & \frac{p\left(S_{n-1}, S_{m-1}\right)}{p\left(S_{n}, S_{m}\right)} e^{\left[p\left(S_{n-1}, S_{m-1}\right)-p\left(S_{n}, S_{m}\right)\right]} \\
= & \frac{\max \left\{S_{n-1}, S_{m-1}\right\}^{k}+\left|S_{n-1}-S_{m-1}\right|}{\max \left\{S_{n}, S_{m}\right\}^{k}+\left|S_{n}-S_{m}\right|} \\
& \cdot e^{\left[\max \left\{S_{n-1}, S_{m-1}\right\}^{k}+\left|S_{n-1}-S_{m-1}\right|-\max \left\{S_{n}, S_{m}\right\}^{k}-\left|S_{n}-S_{m}\right|\right]} \\
= & \frac{S_{m-1}^{k}+\left(S_{m-1}-S_{n-1}\right)}{S_{m}^{k}+\left(S_{m}-S_{n}\right)} \\
& \cdot e^{\left[S_{m-1}^{k}+\left(S_{m-1}-S_{n-1}\right)-S_{m}^{k}-\left(S_{m}-S_{n}\right)\right]} .
\end{aligned}
$$

On the other hand,

$$
\begin{aligned}
S_{m}^{k}+ & \left(S_{m}-S_{n}\right) \\
= & {[1+2+\cdots+m]^{k} } \\
& +[(n+1)+(n+2)+\cdots+(m-1)+m] \\
> & {[1+2+\cdots+m-1]^{k}+m } \\
& +[(n+1)+(n+2)+\cdots+(m-1)+m] \\
> & {[1+2+\cdots+m-1]^{k}+m } \\
& +[(n+1)+(n+2)+\cdots+(m-1)+n] \\
= & S_{m-1}^{p}+\left(S_{m-1}-S_{n-1}\right)+m .
\end{aligned}
$$

Hence, we get

$$
\frac{p\left(T S_{n}, T S_{m}\right)}{p\left(S_{n}, S_{m}\right)} e^{p\left(T S_{n}, T S_{m}\right)-p\left(S_{n}, S_{m}\right)}<e^{-1} .
$$

Therefore $T$ is an $F$-contraction satisfying the conditions of Theorem 21, $\tau=1$, and $T S_{0}=S_{0}$; that is, $S_{0}$ is the unique fixed point of $T$.

\section{Conflict of Interests}

The authors declare that they have no competing interests.

\section{Authors' Contribution}

All authors contributed equally and significantly in writing this paper. All authors read and approved the final paper.

\section{Acknowledgments}

This research was supported by Deanship of Scientific Research (DSR), King Abdulaziz University, Jeddah, Saudi Arabia. The authors thank anonymous referees for their remarkable comments, suggestions, and ideas that helped to improve this paper.

\section{References}

[1] S. G. Matthews, "Partial metric topology," Annals of the New York Academy of Sciences, vol. 728, pp. 183-197, 1994, Proceedings of the 8th Summer Conference on General Topology and Application.

[2] S. G. Matthews, "Partial metric topology," Research Report 212, Department of Computer Science, University of Warwick, 1992.

[3] T. Abdeljawad, E. Karapınar, and K. Taş, "Existence and uniqueness of a common fixed point on partial metric spaces," Applied Mathematics Letters, vol. 24, no. 11, pp. 1900-1904, 2011.

[4] E. Karapınar and İ. M. Erhan, "Fixed point theorems for operators on partial metric spaces," Applied Mathematics Letters, vol. 24, no. 11, pp. 1894-1899, 2011.

[5] E. Karapinar, "Weak $\phi$-contraction on partial contraction," Journal of Computational Analysis and Applications, vol. 14, no. 2, pp. 206-210, 2012.

[6] E. Karapınar, I. M. Erhan, and A. Y. Ulus, "Fixed point theorem for cyclic maps on partial metric spaces," Applied Mathematics \& Information Sciences, vol. 6, no. 1, pp. 239-244, 2012.

[7] E. Karapinar and B. Samet, "Generalized $\alpha-\psi$ contractive type mappings and related fixed point theorems with applications," Abstract and Applied Analysis, vol. 2012, Article ID 793486, 17 pages, 2012.

[8] H. Aydi, E. Karapınar,, and C. Vetro, "On Ekeland's variational principle in partial metric spaces," Applied Mathematics \& Information Sciences, vol. 9, no. 1, pp. 257-262, 2015.

[9] I. A. Rus, "Fixed point theory in partial metric spaces," Analele Universitattii de Vest, Timitsoara, vol. 46, no. 2, pp. 149-160, 2008.

[10] S. Romaguera, "A Kirk type characterization of completeness for partial metric spaces," Fixed Point Theory and Applications, vol. 2010, Article ID 493298, 6 pages, 2010.

[11] L. Ćirić, B. Samet, H. Aydi, and C. Vetro, "Common fixed points of generalized contractions on partial metric spaces and an application," Applied Mathematics and Computation, vol. 218, no. 6, pp. 2398-2406, 2011.

[12] M. Bukatin, R. Kopperman, S. Matthews, and H. Pajoohesh, "Partial metric spaces," American Mathematical Monthly, vol. 116 , no. 8, pp. 708-718, 2009.

[13] P. Hitzler, Generalized metrics and topology in logic programming semantics [Ph.D. thesis], School of Mathematics, Applied Mathematics and Statistics, National University Ireland, University College Cork, 2001.

[14] A. Amini-Harandi, "Metric-like spaces, partial metric spaces and fixed points," Fixed Point Theory and Applications, vol. 2012, article 204, 2012.

[15] E. Karapınar and P. Salimi, "Dislocated metric space to metric spaces with some fixed point theorems," Fixed Point Theory and Applications, vol. 2013, article 222, 2013.

[16] D. Wardowski, "Fixed points of a new type of contractive mappings in complete metric spaces," Fixed Point Theory and Applications, vol. 2012, article 94, 2012. 


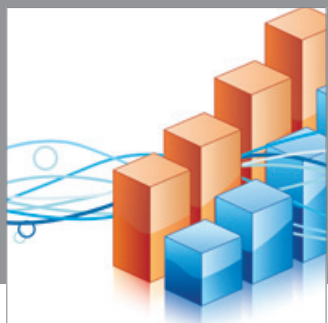

Advances in

Operations Research

mansans

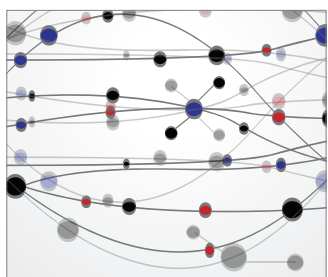

The Scientific World Journal
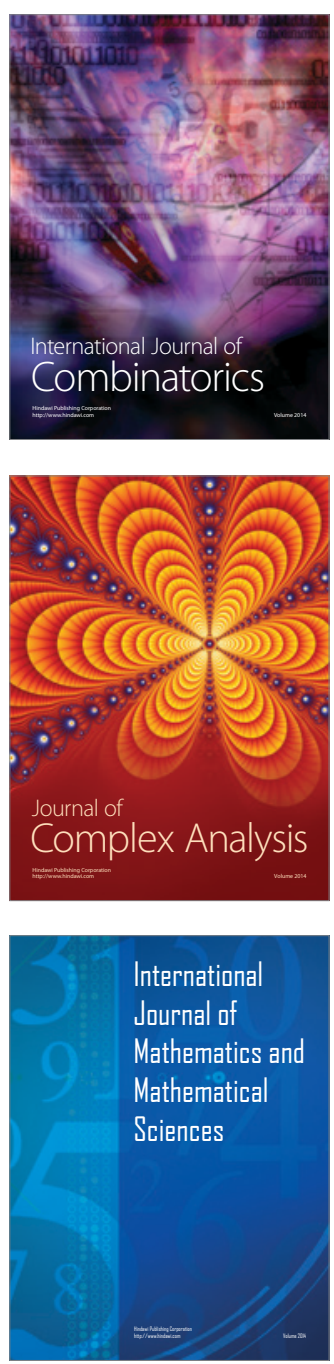
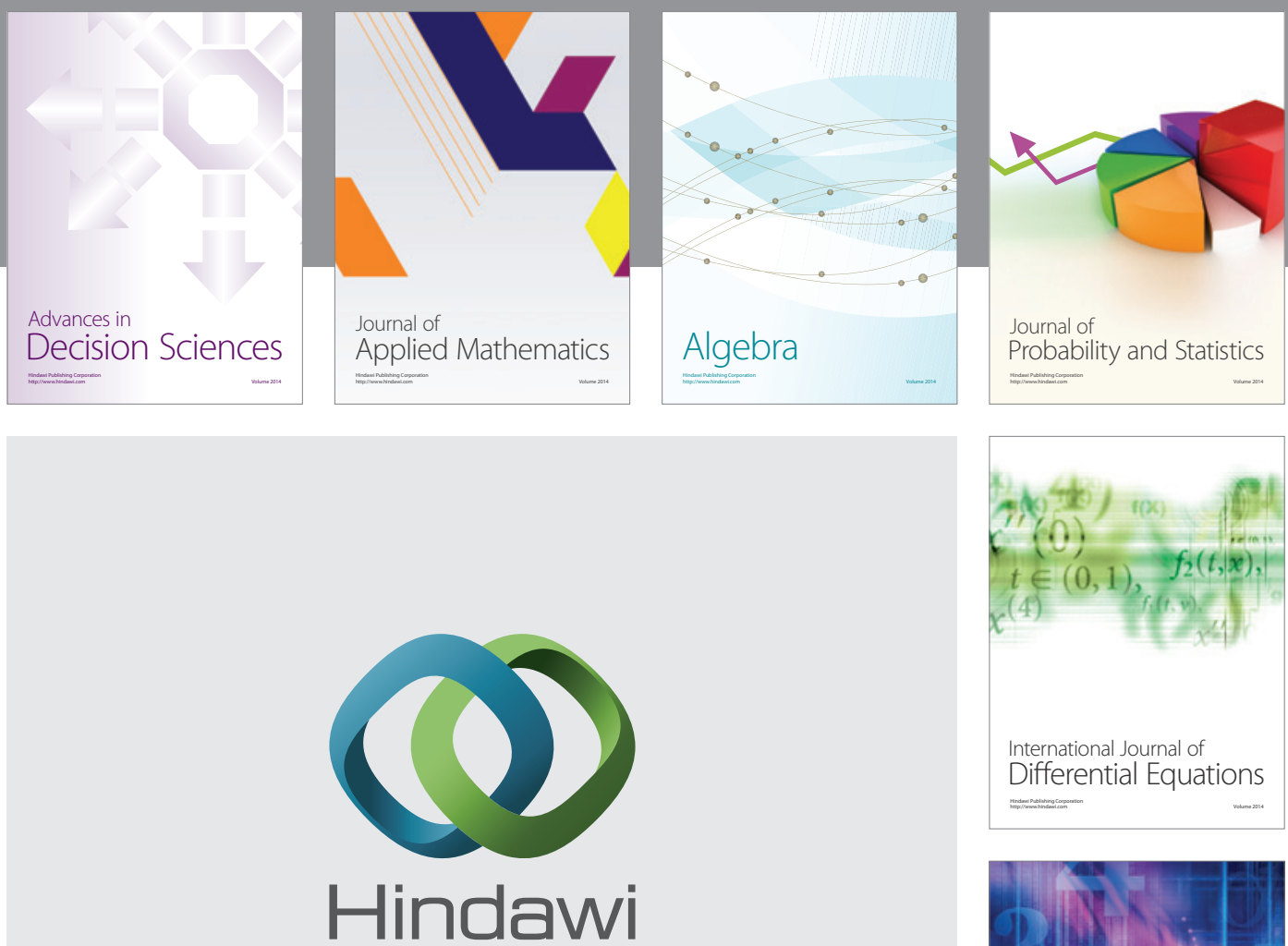

Submit your manuscripts at http://www.hindawi.com
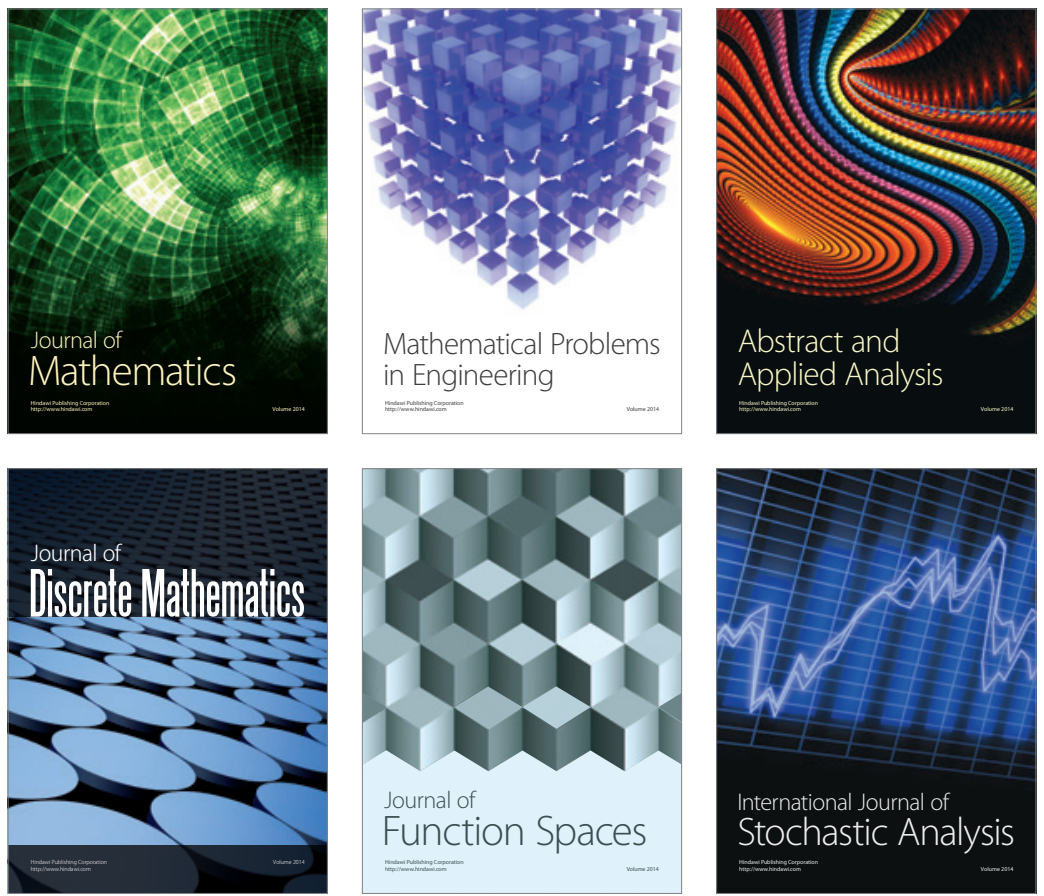

Journal of

Function Spaces

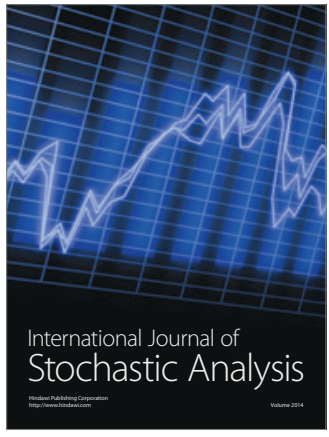

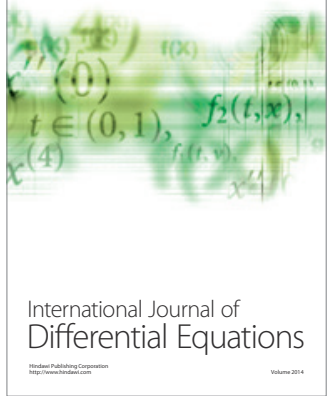
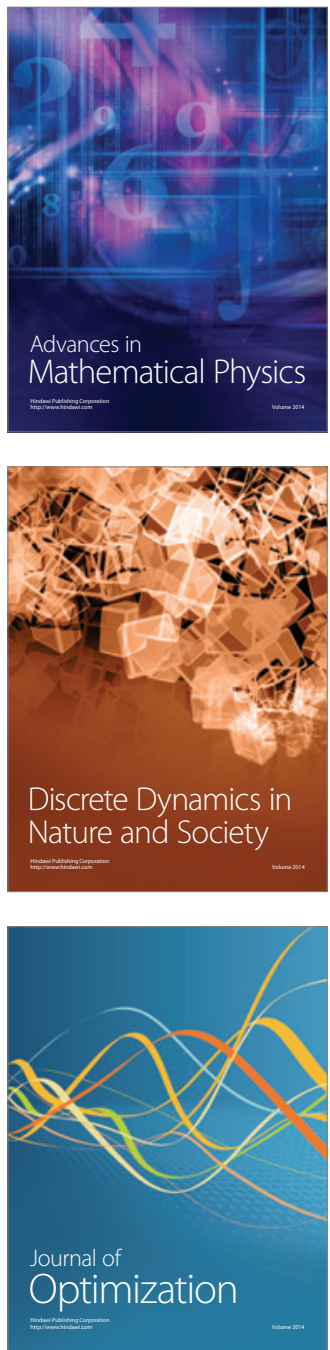